\title{
Masculinização da Tilápia do Nilo, Oreochromis niloticus, Utilizando Diferentes Rações e Diferentes Doses de $17 \alpha$-Metiltestosterona
}

\section{Cleide Schmidt Romeiro Mainardes-Pinto', Nelsy Fenerich-Verani², Benedito Espírito Santo de Campos $^{3}$, Alexandre Livramento da Silva ${ }^{1}$}

\begin{abstract}
RESUMO - Os objetivos deste estudo foram comparar a eficiência de duas rações 1 (NUTRAVIT) e 2 (IP), ambas com $40 \%$ de proteína bruta, contendo o andrógeno sintético $17 \alpha$-metiltestosterona (MT), e analisar a dosagem mais efetiva desse hormônio na reversão sexual da Tilápia do Nilo, Oreochromis niloticus, por intermédio da análise histológica das gonadas e da sexagem dos exemplares. Foram utilizadas 9600 larvas de tilápia do Nilo, com 7 dias de vida, recebendo os seguintes tratamentos, por um período de 45 dias: A - $30 \mathrm{mg} \mathrm{MT/kg} \mathrm{de} \mathrm{ração} \mathrm{1;} \mathrm{B} \mathrm{-} 60 \mathrm{mg}$ MT/kg de ração 1; C - $30 \mathrm{mg}$ MT/kg ração 2; D - $60 \mathrm{mg}$ MT/kg ração 2 e dois grupos controle: E e F, rações 1 e 2, respectivamente, sem hormônio. Os resultados dos testes qui-quadrado das frequiências de indivíduos machos e fêmeas analisados histológica e macroscopicamente mostram que o número de machos obtidos nos tratamentos A, B, C e D foi maior que o dos grupos controle e a dosagem de $60 \mathrm{mg} \mathrm{MT} / \mathrm{kg}$ de ração, nas rações 1 e 2, foi mais eficiente, resultando em $98 \%$ de machos. Entre as rações, o tratamento com a ração 1 apresentou o melhor resultado, para a dosagem de $30 \mathrm{mg} \mathrm{MT} / \mathrm{kg}$ de ração, não havendo, pois, diferença entre rações para a dose de $60 \mathrm{mg} \mathrm{MT/kg} \mathrm{de} \mathrm{ração.}$
\end{abstract}

Palavras-chave: Metiltestosterona, Oreochromis niloticus, reversão sexual, sexagem

\section{Masculinization of Nile Tilapia, Oreochromis niloticus, Using Different Diets and Different Doses of $17 \alpha$-Methyltestosterone}

\begin{abstract}
The objectives of the present study were to compare the efficiency of two diets: 1 (NUTRAVIT) and 2 (IP), both with $40 \%$ of crude protein, containing the synthetic androgen hormone $17 \alpha$-methyltestosterone (MT) and to analyze the most effective dose of this hormone on the sex reversal of Nile tilapia Oreochromis niloticus, throughout histological analysis of the gonads and sex determination of the samples. A total of 9600 Nile tilapia fries with seven days posthatching received the following treatments, per 45 days period: A- $30 \mathrm{mg}$ MT/kg diet 1; B- $60 \mathrm{mg} \mathrm{MT} / \mathrm{kg}$ diet 1; C- $30 \mathrm{mg} \mathrm{MT} / \mathrm{kg}$ diet 2; D- $60 \mathrm{mg} \mathrm{MT} / \mathrm{kg}$ diet 2 and two control groups, $\mathrm{E}$ and $\mathrm{F}$ with diets 1 and 2 hormone free, respectively. The results of chi-square tests of the frequency data of males and females after the treatments and of analysis of histological and macroscopic characteristics showed that the numbers of males obtained by A, B, C and D treatments was higher than the control groups and the dose of $60 \mathrm{mg} \mathrm{MT} / \mathrm{kg}$ of diet, as for the diets 1 and 2, was more efficient, resulting in $98 \%$ of males. Among diets, the treatment A with diet 1 was better than the treatment $\mathrm{C}$ with diet two, for the dose of 30 $\mathrm{mg} \mathrm{MT} / \mathrm{kg}$ of diet. There was no difference among diets for the dose of $60 \mathrm{mg} \mathrm{MT} / \mathrm{kg}$ of the diet.
\end{abstract}

Key Words: methyltestosterone, Oreochromis niloticus, sex-reversal, sex-classification

\section{Introdução}

A rusticidade, a produtividade, o rendimento em filé e a qualidade da carne da tilápia do Nilo, Oreochromis niloticus, contribuíram para despertar o interesse pelo cultivo (FAO, 1987). Porém, o controle reprodutivo é o maior desafio no cultivo dessa espécie, pois a maturação precoce e a reprodução descontrolada levam ao superpovoamento dos tanques, implicando em competição por espaço e alimento, ocasionando baixo crescimento e heterogeneidade no tamanho, dificultando uma criação mais racional e produtiva (WOHLFARTH e HULATA, 1981; MACINTOSH et al., 1985).

Entre as diversas técnicas empregadas para o controle reprodutivo dessa espécie, destacam-se sexagem manual (DUNHAM, 1990), policultivo com espécies piscívoras (LOVSHIN e DA SILVA, 1975), monossexo por hibridação (WOLFARTHe HULATA, 1981), poliploidia (DIAZ e MALDONADO, 1994), ginogênese e androgênese (THORGAARD, 1983) e reversão sexual com a utilização de hormônios (GUERRERO, 1975, 1996; GERRERO III e GUERRERO, 1997; SHELTON et al., 1978;

\footnotetext{
1 Instituto de Pesca - SAA - Núcleo de Aquicultura de Pindamonhangaba - Cx. Postal 151 - CEP: 12400-000. E.mail: ippinda@iconet.com.br 2 Depto. Hidrobiologia UFSCar - Via Washington Luiz, Km 235 - Cx. Postal 676 - São Carlos - SP - CEP: 13565-905. E.mail:verani@power.ufscar.br 3 Instituto de Zootecnia/SAA - Rua Heitor Penteado 56 - CP 60 - Nova Odessa - SP.
} 
NAKAMURA, 1975; TAYAMEN e SHELTON, 1978; PEZZATO et al., 1986; CASTAGNOLLI et al., 1992; e APPEL e LEBOUTE, 1995).

Segundo PHELPS e CEREZO (1992), a utilização de hormônios masculinizantes é a técnica mais prática e efetiva para a produção de machos fenotípicos. Esta prática, além de eliminar problemas relativos à reprodução, proporciona a obtenção de populações constituídas apenas por machos com maior potencial de crescimento. Entre os hormônios pesquisados, o andrógeno sintético $17 \alpha$-metiltestosterona (MT) tem sido bastante empregado no processo de reversão sexual, por apresentar também a vantagem de ser facilmente excretado logo após o período do tratamento hormonal (ROTHBARD et al., 1990; CURTIS et al., 1991; POPMA e GREEN, 1990; e GUERRERO III e GUERRERO, 1997).

Apesar do grande potencial para exploração em um sistema de criação em escala industrial, a tilápia tem sido pouco utilizada, principalmente, devido à baixa disponibilidade de alevinos monossexo e de boa qualidade.

Dessa forma, a possibilidade de se produzir alta porcentagem de alevinos machos, com a utilização de hormônios esteróides, pode se tornar uma forma de aprimorar e disseminar o cultivo dessa espécie.

Os objetivos deste trabalho foram comparar a eficiência de duas rações contendo o (MT) e avaliar a dosagem mais efetiva desse hormônio na reversão sexual da tilápia do Nilo, por intermédio da análise histológica das gonadas e da sexagem dos exemplares.

\section{Material e Métodos}

Este experimento foi conduzido na Estação Experimental de Piscicultura do Instituto de Pesca em Pindamonhangaba-SP, no período de novembro de 1996 a maio de 1997.

Foram utilizadas larvas de tilápia do Nilo, Oreochromis niloticus, coletadas logo após o aparecimento de "ninhadas", ou seja, com aproximadamente 7 dias de vida, em um tanque de acasalamento de reprodutores. Foi feita uma estimativa do número, peso e comprimento médio dessas larvas, por meio do procedimento com base na comparação visual contra um conhecimento padrão (metodologia descrita por POPMA e GREEN, 1990). As larvas com comprimento total médio de $9,1 \mathrm{~mm}$ e peso total médio de $0,012 \mathrm{~g}$ foram distribuídas em seis tanques de alvenaria de $16 \mathrm{~m}^{2}$ cada um, com vazão constante de $20 \mathrm{~L} /$ minuto, na densidade de 100 larvas $/ \mathrm{m}^{2}$ (1.600 indivíduos por tanque) recebendo os seguintes tratamentos:

\begin{tabular}{|c|c|}
\hline \multirow[t]{2}{*}{$\begin{array}{l}\text { Tratamentos } \\
\text { Treatments }\end{array}$} & $\begin{array}{l}\text { Doses hormonais } \\
\text { e rações utilizadas }\end{array}$ \\
\hline & Hormonal doses and used diets \\
\hline $\mathrm{A}$ & 30 mg MT1/kg ração $1 *$ \\
\hline B & 60 mg MT/kg ração 1 \\
\hline $\mathrm{C}$ & $30 \mathrm{mg}$ MT/kg ração $2 * *$ \\
\hline $\mathrm{D}$ & 60 mg MT/kg ração 2 \\
\hline $\mathrm{E}$ & controle - ração 1 sem MT \\
\hline $\mathrm{F}$ & controle - ração 2 sem MT \\
\hline $\begin{array}{ll}\text { MT11 }^{1} & \text { Metiltestost } \\
& \text { Ração 1 (NL } \\
& \text { hormônio m } \\
* * & \text { Ração 2 (IP } \\
& \text { Pesca(Estac } \\
& \text { As dosagen } \\
& 500 \mathrm{~mL} \text { d } \\
& \text { separadam } \\
& \text { ambiente pc }\end{array}$ & $\begin{array}{l}\text { Г) - em pó com } 40 \% \text { PB (ração comercial com } \\
\text { zante). } \\
\text { ó com } 40 \% \text { PB, preparada no Instituto de } \\
\text { rimentalde PisciculturadePindamonhangaba). } \\
\text { (30 e } 60 \mathrm{mg} / \mathrm{kg} \text { de ração) foram diluídas em } \\
\text { letílico, e estas soluções, misturadas } \\
\text { g de ração, permanecendo em temperatura } \\
\text { ras. }\end{array}$ \\
\hline
\end{tabular}

As rações foram administradas quatro vezes ao dia $(8,11,14$ e $17 \mathrm{~h})$ por um período de 45 dias, sendo a taxa de arraçoamento, neste período, de $20 \%$ do peso vivo ao dia (POPMA e GREEN, 1990, RANI e MACINTOSH, 1997) e reajustada a intervalos de 14 dias.

Terminada a fase de tratamento hormonal, os animais foram transferidos para tanques de alvenaria de $100 \mathrm{~m}^{2}$ cada, onde permaneceram por mais 135 dias recebendo ração peletizada com $25 \%$ PB e $2400 \mathrm{kcal} / \mathrm{kg}$ de energia metabolizavel, na proporção de $5 \%$ do peso vivo ao dia, administrada duas vezes ao dia (8 e $16 \mathrm{~h}$ ).

Após esse período, 50 exemplares de cada tratamento foram sacrificados, pesados e medidos, tendo suas gônadas retiradas e fixadas em líquido de BOUIN por 24 horas e, em seguida, transferidas para álcool $70 \% \mathrm{e}$ enviadas para o Laboratório de Histologia do Departamento de Hidrobiologia da Universidade Federal de São Carlos, São Carlos - SP. Após técnicas rotineiras para inclusão em parafina, foram obtidos cortes de $5 \mathrm{a} 7 \mu \mathrm{m}$ de espessura e corados pela Hematoxilina-Eosina. Foi feita a determinação do sexo, bem como o acompanhamento do desenvolvimento gonadal dos exemplares analisados. Orestante dos animais foi submetidoà sexagem, por meio do exame da papila genital.

Para análise comparativa entre as freqüências obtidas de machos e fêmeas com a proporção esperada de 1:1, foi aplicado o teste QUI-Quadrado $\left(\chi^{2}\right)$.

Para o acompanhamento das características da água durante o experimento, diariamente foram efetuados os registros da temperatura da água dos tanques, por meio de termômetro de máxima e mínima e duas vezes por semana, realizadas as análises do teor de oxigênio dissolvido pelo método de Winklere do pH da água, por leitura direta em peagômetro DIGIMED. 


\section{Resultados e Discussão}

Constam da Tabela 1 os valores médios de comprimento total e peso das larvas com 7 dias de vida (início do experimento) e dos exemplares machos e fêmeas com 6 meses (final do experimento).

$\mathrm{O}$ resultado da frequiência de machos e fêmeas identificados por intermédio da análise histológica das gonadas é mostrado na Tabela 2.

No final do experimento, constatou-se que $100 \%$ dos machos analisados histologicamente encontravam-se em maturação, evidenciando células germinativas em diferentes fases de desenvolvimento: espermatogônias, espermatócitos primários, espermatócitos secundários, espermátides e espermatozóides. As fêmeas com a mesma idade, que tiveram suas gonadas analisadas, encontravamse em estádios de repouso e início de maturação.

A Tabela 3 mostra a freqüência de machos e fêmeas identificados por sexagem dos exemplares, bem como a taxa de sobrevivência, no final do experimento, para os diferentes tratamentos.

$\mathrm{O}$ crescimento e a taxa de sobrevivência das larvas durante o tratamento hormonal dependem de

Tabela 1 - Valores médios do comprimento total $\left(\overline{\mathrm{L}}_{t}\right)$ e peso $\left(\overline{\mathrm{W}}_{t}\right)$ das larvas no início do experimento e dos exemplares machos e fêmeas, ao final do experimento, para os diferentes tratamentos

Table 1 - Average values of total length $\left(\overline{\mathrm{L}}_{T}\right.$ and weight $\left.\overline{\mathrm{W}}_{T}\right)$ of O. niloticus fry in the initial period of culture and of the O. niloticus males and females after 6 months of culture

\begin{tabular}{|c|c|c|c|c|c|c|}
\hline \multirow[t]{2}{*}{$\begin{array}{l}\text { Tratamento } \\
\text { Treatment }\end{array}$} & \multicolumn{2}{|c|}{$\begin{array}{l}\text { Larva } \\
\text { Fry }\end{array}$} & \multicolumn{2}{|c|}{$\begin{array}{c}\text { Macho } \\
\text { Male }\end{array}$} & \multicolumn{2}{|c|}{$\begin{array}{l}\text { Fêmea } \\
\text { Female }\end{array}$} \\
\hline & $\begin{array}{l}\overline{\mathrm{L}}_{\mathrm{T}} \text { inicial } \\
\text { Initial } \overline{\mathrm{L}}_{T} \\
\quad(\mathrm{~cm})\end{array}$ & $\begin{array}{l}\overline{\mathrm{W}}_{\mathrm{T}} \text { inicial } \\
\text { Initial } \overline{\mathrm{W}}_{T} \\
\quad(\mathrm{~g})\end{array}$ & $\begin{array}{l}\overline{\mathrm{L}}_{\mathrm{T}} \text { final } \\
\text { Final } \overline{\mathrm{L}}_{T} \\
\quad(\mathrm{~cm})\end{array}$ & $\begin{array}{l}\overline{\mathrm{W}}_{\mathrm{T}} \text { final } \\
\text { Final } \overline{\mathrm{W}}_{T} \\
\quad(\mathrm{~g})\end{array}$ & $\begin{array}{l}\overline{\mathrm{L}}_{\mathrm{T}} \text { final } \\
\text { Final } \overline{\mathrm{L}}_{T} \\
\quad(\mathrm{~cm})\end{array}$ & $\begin{array}{l}\overline{\mathrm{W}}_{\mathrm{T}} \text { final } \\
\text { Final } \overline{\mathrm{W}}_{T} \\
\quad(\mathrm{~g})\end{array}$ \\
\hline $\mathrm{A}$ & 0,90 & 0,012 & 14,33 & 65,00 & 12,30 & 41,30 \\
\hline B & 0,85 & 0,011 & 14,81 & 67,60 & 12,40 & 41,93 \\
\hline $\mathrm{C}$ & 0,90 & 0,011 & 16,20 & 98,00 & 11,80 & 40,20 \\
\hline D & 0,95 & 0,012 & 14,26 & 63,80 & 12,30 & 41,30 \\
\hline $\mathrm{E}$ & 0,92 & 0,013 & 15,36 & 83,00 & 13,30 & 51,00 \\
\hline $\mathrm{F}$ & 0,95 & 0,012 & 14,50 & 66,00 & 12,35 & 41,80 \\
\hline \multicolumn{7}{|c|}{$\begin{array}{l}\mathrm{A}=30 \mathrm{mg} \mathrm{MT} / \mathrm{kg} \text { ração (diet) NUTRAVIT. } \\
\mathrm{B}=60 \mathrm{mg} \mathrm{MT} / \mathrm{kg} \text { ração (diet) NUTRAVIT. } \\
\mathrm{C}=30 \mathrm{mg} \mathrm{MT} / \mathrm{kg} \text { ração (diet) IP. } \\
\mathrm{D}=60 \mathrm{mg} \mathrm{MT} / \mathrm{kg} \text { ração (diet) IP. } \\
\mathrm{E}=\text { controle }- \text { ração NUTRAVIT sem hormô } \\
\mathrm{F}=\text { controle }- \text { ração IP sem hormônio (contr }\end{array}$} \\
\hline
\end{tabular}

Tabela 2 - Freqüência de machos e fêmeas identificados pela análise histológica das gônadas aos 6 meses de vida

Table 2 - Frequency of 0 . niloticus males and females identified by histological analyses of the gonads after 6 months of culture

\begin{tabular}{|c|c|c|c|c|c|}
\hline \multirow{2}{*}{$\begin{array}{l}\text { Tratamento } \\
\text { Treatment }\end{array}$} & \multirow{2}{*}{$\begin{array}{c}\text { № de indíviduos } \\
\text { analisados } \\
\text { Number of analyzed fish }\end{array}$} & \multicolumn{2}{|c|}{$\begin{array}{l}\text { Macho } \\
\text { Male }\end{array}$} & \multicolumn{2}{|c|}{$\begin{array}{l}\text { Fêmea } \\
\text { Female }\end{array}$} \\
\hline & & $\mathrm{N}$ & $\%$ & $\mathrm{~N}$ & $\%$ \\
\hline A & 50 & 44 & 88 & 6 & 12 \\
\hline B & 50 & 50 & 100 & - & - \\
\hline $\mathrm{C}$ & 50 & 42 & 84 & 8 & 16 \\
\hline D & 50 & 48 & 96 & 2 & 4 \\
\hline E & 50 & 32 & 64 & 18 & 36 \\
\hline $\mathrm{F}$ & 50 & 31 & 62 & 19 & 38 \\
\hline
\end{tabular}

$\mathrm{A}=30 \mathrm{mg} \mathrm{MT} / \mathrm{kg}$ ração (diet) NUTRAVIT.

$\mathrm{B}=60 \mathrm{mg} \mathrm{MT} / \mathrm{kg}$ ração (diet) NUTRAVIT.

$\mathrm{C}=30 \mathrm{mg} \mathrm{MT} / \mathrm{kg}$ ração (diet) IP.

$\mathrm{D}=60 \mathrm{mg} \mathrm{MT} / \mathrm{kg}$ ração (diet) $\mathrm{IP}$

$\mathrm{E}=$ controle - ração NUTRAVIT sem hormônio (control diet NUTRAVIT without hormone).

$\mathrm{F}=$ controle - ração IP sem hormônio (control diet-IP without hormone). 
MAINARDES-PINTO et al.

Tabela 3 - Taxa de sobrevivência (\%) e freqüência de machos e fêmeas no final do experimento Table 3 - Survival rate and frequency of males and females in the final of culture

\begin{tabular}{|c|c|c|c|c|c|c|c|}
\hline $\begin{array}{l}\text { Tratamento } \\
\text { Treatment }\end{array}$ & $\begin{array}{c}\text { №1 } \\
\text { inicial } \\
\text { Initial number }\end{array}$ & $\begin{array}{c}\text { №1 } \\
\text { final } \\
\text { Final number }\end{array}$ & $\begin{array}{c}\text { Taxa de } \\
\text { sobrevivência } \\
\text { Survival rate }\end{array}$ & & & & \\
\hline A & 1600 & 960 & 60 & 885 & 92 & 75 & 8 \\
\hline B & 1600 & 1120 & 70 & 1108 & 99 & 12 & 1 \\
\hline $\mathrm{C}$ & 1600 & 1040 & 65 & 926 & 89 & 114 & 11 \\
\hline D & 1600 & 1088 & 68 & 1066 & 98 & 22 & 2 \\
\hline $\mathrm{E}$ & 1600 & 1040 & 65 & 605 & 58 & 435 & 42 \\
\hline $\mathrm{F}$ & 1600 & 1120 & 70 & 630 & 56 & 490 & 44 \\
\hline
\end{tabular}

$\mathrm{N}^{1}=$ número de exemplares.

$N^{1}=$ number of fishes.

$\mathrm{A}=30 \mathrm{mg} \mathrm{MT} / \mathrm{kg}$ ração (diet) NUTRAVIT.

$\mathrm{B}=60 \mathrm{mg} \mathrm{MT} / \mathrm{kg}$ ração (diet) NUTRAVIT

$\mathrm{C}=30 \mathrm{mg} \mathrm{MT} / \mathrm{kg}$ ração (diet) IP.

$\mathrm{D}=60 \mathrm{mg} \mathrm{MT} / \mathrm{kg}$ ração (diet) IP.

$\mathrm{E}=$ controle - ração NUTRAVIT sem hormônio (control diet NUTRAVIT without hormone).

$\mathrm{F}=$ controle - ração IP sem hormônio (control diet-IP without hormone).

vários fatores, entre eles densidade de estocagem, alimentação e temperatura (BOCEK et al., 1992).

Neste experimento, não houve diferença quanto ao ganho de peso e à taxa de sobrevivência entre os tratamentos, corroborando os resultados obtidos por VERA CRUZ E MAIR (1994) e GUERRERO III e GUERRERO (1997), os quais afirmaram que o metiltestosterona tem pouco ou nenhum efeito sobre o crescimento e a sobrevivência de tilápia do Nilo durante o tratamento hormonal.

A mortalidade observada durante a fase de tratamento hormonal pode ser explicada pelo estabelecimento de hierarquia na alimentação entre os peixes. Indivíduos dominantes dentro de uma população podem consumir mais alimento e crescerem mais rapidamente, deixando menos alimento para os indivíduos submissos que apresentam menor crescimento e tornam-se, conseqüentemente, vulneráveis ao canibalismo (VERA CRUZ e MAIR, 1994).

Os resultados do teste de $\chi^{2}$ aplicados entre as freqüências de indivíduos machos e fêmeas analisados macroscopicamente mostram que:

- o número de machos obtidos nos tratamentos A, B, C e D foi significativamente maior $(\mathrm{P}<0,01)$ que o dos grupos controle $\mathrm{E}$ e $\mathrm{F}$.

- a dosagem de $60 \mathrm{mg} \mathrm{MT/kg} \mathrm{de} \mathrm{ração,} \mathrm{tanto}$ NUTRAVIT, quanto IP, foi mais eficiente, resultando em maior número de machos $(\mathrm{P}<0,01)$.

Verificou-se que o tratamento com a ração NUTRAVIT foi significativamente melhor $(\mathrm{P}<0,05)$ que o tratamento com ração IP, para a dosagem de $30 \mathrm{mg} \mathrm{MT/kg} \mathrm{de} \mathrm{ração.}$

Pela análise das Tabelas 2 e 3, conclui-se que a identificação de machos e fêmeas por sexagem é possível e confiável, pois os resultados em termos de frequência foram semelhantes aos obtidos pela análise histológica das gônadas.

Durante o tratamento hormonal, a temperatura média da água dos tanques foi de $26^{\circ} \mathrm{C}$, estando dentro da faixa considerada ideal para o processo de reversão sexual (SHELTON et al. 1978). Os valores do $\mathrm{pH}$ variaram de 6,8 a 8,5 e os de oxigênio dissolvido, de 5,5 ppm a $10,0 \mathrm{ppm}$, sendo considerados normais para piscicultura (BOYD e SCARSBROOK, 1974).

BOCEK et al. 1992 e PHELPS et al. (1995) obtiveram, respectivamente, 97 e $94,3 \%$ de machos utilizando as mesmas dosagens dehormônio, quantidade e frequência de arraçoamento que as utilizadas neste experimento.

Segundo PONZA et al. (1996), diversas variáveis podem influir no processo da reversão sexual, por meio da utilização do metiltestosterona, incluindo o ambiente de desenvolvimento das larvas, a dosagem e a duração do tratamento hormonal, o tipo, a quantidade e a frequência da alimentação.

Vários autores utilizando dietas com metiltestosterona fornecidas duas a quatro vezes ao dia obtiveram populações masculinas entre 95 e $99 \%$, raramente $100 \%$, devido à aparente resistência ao hormônio por 1 a $5 \%$ das fêmeas tratadas (MACINTOSH et al., 1988; BOCEK et al., 1992; e PHELPS et al., 1995).

No presente estudo, a freqüência de arraçoamento utilizada foi de quatro vezes ao dia, resultando em 98\% de indivíduos machos.

Segundo GUERRERO III e GUERRERO, (1997) a frequiência do arraçoamento durante o período do tratamento hormonal, quando é de cinco a seis vezes 
ao dia, resulta em maior quantidade de machos revertidos. Dessa forma, RANI e MACINTOSH (1997) obtiveram $100 \%$ de machos arraçoando as larvas seis vezes ao dia durante o tratamento hormonal.

\section{Conclusões}

A dosagem de $60 \mathrm{mg}$ de metiltestosterona, independente do tipo da ração utilizada no experimento, foi a mais eficiente, obtendo-se $98 \%$ de machos identificados tanto histológica quanto macroscopicamente.

Entre as rações testadas, a ração 1 apresentou melhores resultados quanto ao efeito da masculinização.

Outras pesquisas deverão ser feitas utilizando outras doses de hormônio e outras freqüências de arraçoamento.

\section{Agradecimento}

À Dra ${ }^{\mathrm{a}}$. Massuka Y. Narahara, pelas críticas e sugestões.

À Técnica de Apoio à Pesquisa Maria Inês C. Manoel, pela digitação do texto, e aos funcionários José Menino Corrêa, José Chavone e João Roberto Rezende, pela colaboração durante as etapas do trabalho de campo.

\section{Referências Bibliográficas}

APPEL, H.B., LEBOUTE, E.M. Masculinização de pós-larvas de tilápia do Nilo (Oreochromis niloticus) utilizando andrógenos através de tratamento de imersão. In: ENCONTRO RIOGRANDENSE DE TÉCNICOS EM AQUICULTURA, 3, Ibirubá, RS, 1995. Anais...Ibirubá, 1995, p.113-119.

BOCEK, A., PHELPS, R.P., POPMA, T.J. 1992. Effect of feeding frequency on Sex-reversal and on growth of Nilo tilapia, Oreochromis niloticus. J. Appl. Aquac., 1(3):97-103.

BOYD, C.E., SCARSBROOK, E. 1974 Effects of agricultural limestone on phytoplancton communities of fish ponds. Arch. Hydrobiology, 74(3):336-349.

CASTAGNOLLI, N., LEME DOS SANTOS, H.S., FONSECA, S.S. Reversão sexual em tilápia do Nilo (Oreochromis niloticus) através do uso do hormônio undecanato de testosterona na ração. In: ENCONTRO NACIONAL DE AQUICULTURA - SIMPÓSIO BRASILEIRO DE AQUICULTURA VII e ENCONTRO BRASILEIRO DE PATOLOGIA DE ORGANISMOS AQUÁTICOS, 2, Peruíbe, SP, 1992. Resumos...Peruíbe, 1992, p.39.

CURTIS, L.R., DIREN, F.T., HURLEY, M.D. et al. 1991. Disposition and elimination of 17 a-metiltestosterone in Nile tilapia (Oreochromis niloticus). Aquaculture, 99:193-201.

DIAZ, M. 1994. Análisis de viabilidad y crecimiento hasta el levante de triploides y diploides de tilapia nilotica (Oreochromis niloticus, Linné). Boletim Científico INPA n.2, p.33-45.
DUNHAN, R.A. 1990. Production and use of monosex or sterile fishes in aquaculture. Rev. Aquac. Sci., 2(1):1-17.

FAO. 1987. Yearbook of fishery statistics - secis. Catches and Landings. FAO, Rome, v.64, p.141.

GUERREIRO III, R.D. 1975. Use of androgens for production of all-male Tilapia aurea (Steindachner) Transaction of American Fisheries Society, 104(2):342-348.

GUERRERO III, R.D. 1996. Aquaculture in the Philippines. World Aquac., 27(1):7-13.

GUERRERO III, R.D. GUERREIRO L.A. Effects of Androstenedione and methyltestosterone on Oreochromis niloticus Fry treated for sex reversal in outdoor Net Enclosure. In: INTERNATIONAL SYMPOSIUM ON TILAPIA IN AQUACUltURE, 4, Orlando, Florida - USA, 1997. Proceedings... Orlando, v.12, 1997, p.772-777.

LOVSHIN, L., DA SILVA, A.B. Culture of monosex and hibrid tilapias. In: SYMPOSIUM ON AQUACULTURE ON AFRICA, Accra, (s.1.) FAO, CIFA. 16P. 1975.

MACINTOSH, D.J., VARGHESE, T.J., RAO, G.R.S. 1985. Hormonal sex reversal of wild spawned tilapia in India. J. Fish Biology, 26:87-94.

MACINTOSH, D.J., SINGH,T. B., LITTLE, D.C. et al. Growth and sexual development of 17 a-methyltestosterone and progesterone. Treated Nile tilapia, Oreochromis niloticus reared in earthen ponds. In: INTERNATIONAL SYMPOSIUM ON TILAPIA IN AQUACULTURE, 15, Bangkok, Thailand, 1988. Proceedings... Bangkok, Thailand, 1988, p.457-463.

NAKAMURA, M. 1975. Dosage-dependent changes in the effect of oral administration of methyltestosterone on gonadal differentiation in Tilapia mossambica. Bulletin of the Faculty of Fisheries, 26(2):99-108.

PEZZATO, L.E., PACHKER, I.U., FORESTI, F. et al. Ação de hormônios sexuais sobre larvas da tilápia do Nilo (Oreochromis niloticus) durante a fase de reversão sexual, mortalidade e freqüência de reversão. In: SIMPÓSIO BRASILEIRO DE AQUICULTURA, 4, Cuiabá, MT, 1986. Anais... Cuiabá, 1986, p.63-69.

PHELPS, R.P., CEREZO, G. 1992. The effect of confinemente in hapas on sex reversal and growth of Oreochromis niloticus. J. Appl. Aquac., 1(4):73-81.

PHELPS, R.P., SALAZAR, G.C., ABE, V., ARGUE, B. 1995. Sex reversal and nursery growth of Nile Tilapia, Oreochromis niloticus (L.), free-swimming in earthen ponds. Aquac. Res., 26:293-295.

POPMA, T.J., GREEN, B.W. 1990. Reversão sexual de tilápias em tanques de terra. In: Manual de produção em aquacultura. Flórida - EUA: Universitu Aurburn. 52p.

PONZA, S., LITTLE, D.C., BOOTHAMJINDA, C. 1996. Effects of the timing of treatment on set after hatching on the effecacy of 17 a-methyltestosterone for masculinization of Nile Tilapia (Oreochromis niloticus) fry. In: CRESWELL, R.L. (ed.) World Aquaculture. Book of Abstracts. Lousiania State University, Baton Rouge, USA: World Aquaculture Society. p.314-315.

RANI, A., MACINTOSH, D.J. An evaluation of the effects of hormone concentration, treatment period, feeding regime, and rearing salinity on production of all-male Nile Tilapia (Oreochromis niloticus) fry using 17 a methyltestosterone. In: SYMPOSIUM ON TILLAPIA IN AQUACULTURE, 4, Orlando, Flórida - USA, 1997. Proceedings... Flórida, v.2, p.791-802, 1997.

ROTHBARD, S.W., ZOHAR, Y., ZAMORA, N. et al. 1990. Clearence of 17 apha-methyltestosterone form muscle of 
sex-inversed tilapia hybrids treated for growth enhancment with two doses of the androgen. Aquaculture, 89(3/4):365-376.

SHELTON, W.L., HOPKINS, K.D., JENSEN, G.L. 1978. Use of hormones to produce monosex tilapia for aquaculture. In: SMITHERMAN, R.O. SHELTON, W.L., GROVER, J.H. (Eds) Culture of exotic fishes symposium. Alabama University: Fish Culture Section/ Am. Fish. Soc. Auburn. p.10-33.

TAYAMEN, M.M., SHELTON, W.L. 1978. Inducement of Sex reversal in Sarotherodon niloticus(L.). Aquaculture, 14(4):349-354.

THORGAARD, G.H. 1983. Chromosome set manipulation and sex control in fish. In: HOAR, W.W., RANDALL, D.J., DONALDSON, E.M. (Eds.) Fish physiology. New York: Academic Press. v.9, part B. p.405-428.
VERA CRUZ, E.M., MAIR, G.C. 1994. Conditions for efective androgen sex-reversal in Oreochromis niloticus (L.) Aquaculture. 122(2,3):237-248.

WOHLFARTH, G.W., HULATA, G.I. 1981. Applied genetics of tilapias. ICLARM Studies and Reviews Manila, Philippines, v.6, 26p.

Recebido em: 28/01/99

Aceito em: 05/12/99 